\title{
TV/Series
}

$6 \mid 2014$

Écho et reprise dans les séries télévisées (III) : de la métafiction à la transmédialité

\section{Échos en série : Formes et enjeux de la réplique dans The Wire}

\section{Livio Belloï}

\section{(2) OpenEdition \\ Journals}

Édition électronique

URL : http://journals.openedition.org/tvseries/315

DOI : 10.4000/tvseries.315

ISSN : 2266-0909

Éditeur

GRIC - Groupe de recherche Identités et Cultures

Référence électronique

Livio Belloï, «Échos en série : Formes et enjeux de la réplique dans The Wire », TV/Series [En ligne],

6 | 2014, mis en ligne le 01 décembre 2014, consulté le 19 avril 2019. URL : http://

journals.openedition.org/tvseries/315; DOI : 10.4000/tvseries.315

\section{(c) (i) (9)}

TV/Series est mis à disposition selon les termes de la licence Creative Commons Attribution - Pas d'Utilisation Commerciale - Pas de Modification 4.0 International. 


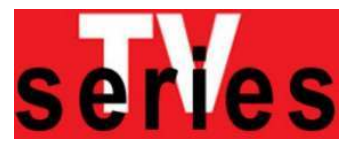

\section{Échos en série : \\ Formes et enjeux de la réplique dans The Wire}

Livio BELLÖ̈

Vaste « roman visuel » (David Simon), la série The Wire (HBO, 2002-2008) se donne pour ambition d'explorer sous toutes ses coutures la ville américaine de Baltimore. En termes de construction narrative, les soixante épisodes composant cette série s'ordonnent à un ample mouvement cyclique, au terme duquel rien, en définitive, n'a vraiment changé : The Wire se clôture en effet par un cinglant retour à la case départ. Au sein de cette structure en boucle, les phénomènes d'écho et de reprise foisonnent et affectent la série sous de multiples aspects. Le présent article s'emploie à dégager divers effets d'écho en tant qu'ils se jouent dans la sphère du langage. Il s'agit plus précisément de porter au jour un curieux microphénomène selon lequel, tout au long de la série, certains personnages sont amenés à prononcer les mêmes lignes de dialogue, tantôt de manière délibérée, tantôt de manière inconsciente. Après avoir débusqué la première occurrence de ce phénomène dans la trame complexe de The Wire, nous nous attacherons à examiner quelques cas de figure précis, dans une perspective qui se veut d'abord morphologique. Chemin faisant, une notion s'impose : celle de réplique, que The Wire, comme objet de langage, nous invite à entendre dans un fécond double sens - ligne de dialogue et fait d'itération.

A

$\mathrm{u}$ terme de l'ultime séquence de The Wire (HBO, 2002-2008), vaste radioscopie de la ville américaine de Baltimore amer, c'est selon, qui s'impose en images au regard désabusé de l'exinspecteur Jimmy McNulty : la grande roue a tourné d'un cran, mais les mécanismes sont restés identiques à eux-mêmes, prêts à entrer de nouveau en action ${ }^{1}$.

Créée par un ex-journaliste (David Simon) et un ex-policier (Ed Burns), The Wire obéit, en termes de structure, à un ample mouvement cyclique, qui constitue l'une des modalités de sa "complexité narrative " (pour reprendre l'expression, devenue fameuse, de Jason Mittell2). Dans la séquence finale de la série, cette structure en boucle se trouve accusée par la reprise d'un thème musical familier à l'oreille du spectateur : il s'agit de Way Down in the Hole, chanson composée

${ }^{1}$ Sur ce point, voir par exemple Marsha Kinder, « Re-Wiring Baltimore: The Emotive Power of Systemics, Seriality, and The City ", in Film Quarterly, Vol. 62, No. 2, hiver 2008-2009, p. 57 [p. 50-57].

2 Jason Mittell, "Narrative Complexity in Contemporary American Television ", in The Velvet Light Trap, No. 58, hiver 2006, p. 29-40. S'agissant de The Wire, les propositions de Mittell ont fait l'objet d'un premier approfondissement par Ted Nannicelli, «It's All Connected: Televisual Narrative Complexity ", in The Wire. Urban Decay and American Television, éd. Tiffany Potter et C. W. Marshall, New York, Londres, Continuum, 2009, p. 190-202. 
par Tom Waits, modulée au fil des différentes saisons ${ }^{3}$, mais ici interprétée par les Blind Boys of Alabama, soit la musique même qui aura ponctué le générique du tout premier épisode de la série. La fin comme retour au début, en quelque sorte, au prix d'un puissant effet de bouclage dans lequel se cristallisent emblématiquement les déterminismes en chaîne que la série aura contribué à porter au jour.

En pareil univers, la reprise occupe, l'on s'en doute, un rôle cardinal. De fait, elle touche la série sous de multiples aspects, du plus circonscrit au plus général, et peut prendre des formes extrêmement diversifiées. La présente étude se bornera à examiner les effets d'écho et de reprise en tant qu'ils se jouent en territoire langagier.

Il y aurait de toute évidence mille choses à dire sur The Wire en tant qu'objet de langage. Ainsi Flore Coulouma veut-elle voir dans cette série une véritable "expérience en matière de linguistique de terrain 4 ». Partant de cette observation, l'auteure s'attache à étudier les tropes et autres stéréotypes qui émaillent les propos tenus par les trafiquants de drogue tout au long de la première saison de The Wire. Dans ces tours de parole où les mêmes expressions fleurissent inlassablement, ne se refusant aucune formulation tautologique (« The game's the game»), Coulouma discerne essentiellement des enjeux d'ordre identitaire et clanique. Dans une autre optique, Mathieu PotteBonneville a, pour sa part, émis l'hypothèse selon laquelle le langage constitue peut-être "le véritable personnage central de The Wire 5 ». D’un point de vue général, Potte-Bonneville croit pouvoir distinguer à cet égard trois grandes fonctions du langage. Sur les trottoirs de Baltimore, le langage peut ainsi prendre valeur, alternativement ou simultanément, de "marqueur d'appartenance», de "vecteur de pouvoir» et d'« opérateur de singularisation». Comme marqueur d'appartenance, le langage a en propre, selon l'auteur, de « retrempe[r] les formes d'un entre-soi où les acteurs trouvent d'abord à se définir, à parler pour dessiner ce qui entre eux va sans dire ${ }^{6} »$. En tant que vecteur de pouvoir, le langage, à suivre Potte-Bonneville, "trace en permanence le diagramme des dominations stratégiques qui s'exercent pour autant qu'elles se disent, mais s'exposent aussi en s'énonçant à se voir déjouées dans l'ironie, renversées dans l'humour ${ }^{7}$ ». À titre d'opérateur de singularisation enfin, le langage « double l'inscription sous les règles communes de la possibilité de s'y faire entendre, et

${ }^{3}$ Sur les différentes versions de cette chanson dans The Wire, véritables variations autour d'un même thème, voir par exemple Rafael Alvarez, The Wire. Truth Be Told, Edimbourg, Londres, New York, Melbourne, Canongate, 2009, p. 246-250.

4 Flore Coulouma, "'That's the game, yo': Stereotype and Identity in The Wire ", in TV/ Series, No. 1, juin 2012, p. 144-162 (p. 144 pour le passage cité).

5 Mathieu Potte-Bonneville, "All in the game ", in The Wire. Reconstitution collective, éd. Emmanuel Burdeau et Nicolas Vieillescazes, Paris, Les Prairies Ordinaires/Capricci, 2011, p. 164 [p. 147-166].

6 Ibid., p. 164-165.

7 Ibid., p. 165. 
jouer, une variation propre, comme un méandre ou un bief dans le flot des paroles échangées ${ }^{8}$ ".

Dans ce qui suit, il s'agira de dégager et d'explorer une possible quatrième fonction du langage, qu'il faudra elle aussi nommer, et qui, dans le registre de la parole, est bel et bien affaire de reprise. Il sera plus précisément question d'un micro-phénomène qui, de prime abord, pourra paraître négligeable, mais qui, tout bien considéré, permet, en raison de son caractère aussi discret que systématique, non seulement d'éclairer l'un des enjeux majeurs de The Wire en tant qu'objet linguistique, mais aussi de déterminer avec plus de précision la nature du regard dont la série tout entière procède.

La première manifestation du phénomène en question se produit très tôt dans la série: elle prend place, en l'occurrence, au début de l'épisode 1.2 («The Detail »). Nous sommes dans le bureau du juge Phelan. Ce dernier écoute distraitement l'inspecteur McNulty, qui lui rapporte qu'un certain William Gant, témoin oculaire dans une affaire de meurtre liée au clan Barksdale, vient de se faire assassiner d'une balle dans la tête. Alors qu'il mange un sandwich, Phelan souille sa cravate d'un peu de moutarde. La conversation, assez tendue, se poursuit, cependant que le juge s'efforce de nettoyer sa cravate à l'aide d'une serviette en papier. À un certain moment, au beau milieu de ses récriminations, McNulty, cadré en plan rapproché à l'épaule, fait incidemment remarquer à Phelan qu'il "a raté un endroit » ("You missed a spot »). Sans autre transition qu'une simple coupe franche, la séquence suivante nous transporte dans le sous-sol désaffecté où officiera désormais la nouvelle équipe dirigée par le lieutenant Cedric Daniels. L'inspecteur Santangelo est occupé à nettoyer le sol à l'aide d'une serpillière. L'un de ses collègues plus âgés (Patrick Mahone) lui fait observer, index tendu et regard moqueur, que, dans son opération de nettoyage, il a " raté un endroit » (« Hey, you missed a spot»). Si l'énoncé est sensiblement le même, il ne véhicule pas des connotations identiques : dans la bouche de McNulty, ces mots résonnent comme une marque d'attention, alors que, du côté de Mahone, il s'agit plutôt de pointer une négligence, sur le ton de la raillerie.

Petit moment de bégaiement apparent de la série, d'autant plus frappant qu'un intervalle très court (moins d'une minute) sépare les deux prises de parole. En termes sémantiques, rien de plus gratuit que la phrase en question : parole triviale, anecdotique, dépourvue de la moindre incidence sur un plan narratif. Mais ce qui importe en la circonstance, c'est sans doute moins l'énoncé en lui-même que son inscription au sein d'une structure d'écho. Paradoxalement, le phénomène se rend d'autant plus manifeste qu'il fait fond sur une parole pauvre, une parole qui, par là, fait d'autant mieux ressortir le mécanisme, le fait même de la répétition.

${ }^{8}$ Ibid. 
Une telle itération n'est évidemment pas sans danger en termes de vraisemblance narrative. D'un côté, la répétition des mêmes lignes de dialogue peut être reçue, par le spectateur, sinon comme une paresse d'écriture, du moins comme une maladresse dans laquelle l'appareil énonciatif de la série se serait fourvoyé. Pire encore, dès lors qu'il se réitère, tout particulièrement dans un laps de temps à ce point réduit, l'énoncé verbal attire potentiellement l'attention sur lui-même ; il tend à se donner pour ce qu'il est, ligne de dialogue articulée par un acteur, et non acte de locution assumé par un personnage. En d'autres termes, la répétition concourt à accuser l'énoncé verbal comme réplique. Dans cette mesure, la réplique, entendue au double sens du terme, tout ensemble élément de dialogue et fait d'itération, porte virtuellement atteinte à l'effet diégétique.

"You missed a spot »- le même énoncé, anodin en apparence, fait retour d'une séquence à l'autre: c'est là un risque, à n'en pas douter, mais, semble-t-il, un risque calculé et pleinement assumé. D'une certaine façon, il ne pouvait en aller autrement. Puisqu'il s'agit en effet d'une première occurrence, le procédé se devait d'être pleinement perceptible - et peu importe l'effet local de doublon ou de redite. Par son office, The Wire invite d'ores et déjà son spectateur à " écouter attentivement » (conformément au leitmotiv de la première saison) et à s'inquiéter de divers phénomènes de reprise affectant la sphère du langage 9 . Dans le cas qui nous occupe, on pourrait parler de reprise in absentia, en ce qu'elle affecte deux lignes narratives isolées, sans que les personnages concernés soient présents l'un à l'autre et sans qu'ils soient en outre conscients des prises de parole assumées par leur homologue ${ }^{10}$. Dans cette occurrence inaugurale, il est par ailleurs à

${ }_{9}$ Cette idée de « rater un endroit » fera du reste retour beaucoup plus tard dans la série, créant presque les conditions d'un running gag en bonne et due forme. Vers le milieu de l'épisode 4.1 («Boys of Summer») en effet, le sergent Carver, accompagné de l'agent Colicchio, rend visite à Bodie et à son équipe de dealers de rue. Tirant prétexte d'une certaine complicité, Carver profite de son passage pour réaffirmer devant Bodie et ses comparses les vertus de la courtoisie élémentaire. Vers le milieu de cette séquence, le dialogue quelque peu sardonique entre Carver et Bodie se suspend et se déplace : le sergent interpelle en effet Lex, l'homme à tout faire de ce coin de rue, occupé à balayer le trottoir. Après les politesses habituelles et convenues, Carver fait observer au jeune homme : «Je ne veux pas critiquer, mais je crois que tu as raté un endroit » ( Not to criticize or anything, but I think you missed a spot there »). Réplique à distance cette fois qui, ironiquement, confère à Lex le même statut que l'inspecteur Santangelo au début de l'épisode 1.2 et qui vaut comme clin d'œil à la mise en œuvre inaugurale du procédé.

${ }^{10}$ À la reprise in absentia, pourrait utilement s'opposer, en termes typologiques, la reprise in praesentia, elle aussi assez abondante dans The Wire. À l'enseigne de la reprise in praesentia, il convient de placer tous les cas de figure impliquant qu'une ou plusieurs lignes de dialogue transitent d'un personnage à l'autre sur le double mode de la répétition consciente et du retour d'ascenseur verbal. Pour ne prendre qu'un exemple, la première saison de The Wire se trouve pour ainsi dire encadrée par un remarquable effet de reprise in praesentia. Au début de l'épisode 1.1, la série nous donne à voir le procès de D'Angelo Barksdale. Deux témoins se succèdent à la barre : William Gant (personnage auquel nous avons déjà fait allusion) et Nakeisha Lyles. Alors que Gant confirme que c'est bien D'Angelo qui est le coupable, Nakeisha Lyles, pour sa part, se rétracte subitement, probablement en 
noter que la réplique n'engage nullement à postuler un rapport d'homologie entre les personnages concernés ; elle correspond plutôt à l'exposition d'un procédé, ici actualisé dans toute sa nudité, sans autre véritable enjeu que lui-même, mais que la série s'attachera, par la suite, à investir sous de multiples formes.

Dans la dernière séquence de l'épisode 3.11 («Middle Ground »), le personnage de Stringer Bell, lieutenant et ami d'enfance du tout-puissant gangster Avon Barksdale, se retrouve en bien fâcheuse posture: non seulement parce que ses investissements immobiliers s'avèrent beaucoup plus coûteux que prévu (comme il vient de s'en ouvrir, non sans courroux, à l'entrepreneur Andrew Krawczyk), mais aussi - et surtout - parce qu'au terme d'une course effrénée dans l'immeuble à appartements qu'il a entrepris de faire rénover, il tombe dans un piège tendu de concert par Omar Little, son ennemi de toujours, et par Brother Mouzone, tueur à gages dont il a trahi les attentes et failli précipiter la perte. Séquence particulièrement tendue que celle-ci, par laquelle se clôture un processus enclenché dès la première saison (avec le meurtre particulièrement sauvage de Brandon, l'amant d'Omar, révélé au début de l'épisode 1.6) et qui apporte un point final à un cycle de vengeances en chaîne, cortège ininterrompu d'assauts et de ripostes ${ }^{11}$.

Mis en joue par ses deux adversaires, Bell n'a d'autre choix que de multiplier les manœuvres dilatoires : en premier lieu, il se plaindra implicitement de l'inégalité du combat, soulignant le fait que, pour sa part, il n'est pas armé («I ain’t strapped »); ensuite, il invoquera un détachement, une prise de distance en regard de sa vie de gangster («I

raison des pressions exercées sur sa personne par le clan Barksdale. McNulty comprend vite la combine et, dépité, quitte la salle d'audience. Au passage, il souffle à Stringer Bell, assis au fond de la salle : « Joli coup » (« Nicely done »). Â l'autre bout de la saison, c'est-à-dire à la fin de l'épisode 1.13 , c'est au procès du clan Barksdale en son ensemble que nous assistons, avec sa panoplie d'arrangements tacites et autres chicanes juridiques. À nouveau déçu par la tournure des événements (notamment par le fait qu'en définitive, D’Angelo, convaincu par sa mère, reste muet comme une carpe quant aux agissements d'Avon Barksdale et de ses complices), McNulty décide d'aller s'asseoir en dehors de la salle d'audience. Accompagné par la mère de D’Angelo, Stringer Bell sort à son tour de la salle et adresse à McNulty la phrase même que celui-ci avait prononcée au début du premier épisode : "Joli coup » ( "Nicely done »). Autour du même énoncé, le destinateur devient destinataire et vice-versa. Notons que la reprise in praesentia joue en l'espèce d'autres effets de symétrie: dans la séquence de l'épisode 1.1, Stringer Bell est assis, alors que McNulty est debout et en mouvement; dans la scène de l'épisode 1.13, c'est exactement le contraire : McNulty est affalé, songeur, sur un banc, cependant que Stringer Bell est pour sa part debout et en mouvement. Cas assez extrême, cette reprise in praesentia se déploie sur l'arc entier d'une saison: elle sollicite fortement et l'attention, et la mémoire du sujet spectatoriel (lequel peut ne pas percevoir du premier coup cet effet de rime à distance). Un tel cas d'espèce devrait inciter à s'interroger sur la portée, en termes temporels, de l'effet de réplique (de séquence à séquence, d'épisode à épisode, de saison à saison).

${ }^{11}$ Pour une généalogie de l'alliance relativement inattendue entre Omar et Mouzone, voir Ted Nannicelli, op. cit., p. 194-195. 
ain't involved, I ain't involved in that gangster bullshit no more ») ; enfin, manœuvre désespérée, il se retranchera derrière un argument financier, proposant de l'argent contre une vie sauve. Mais ni Omar, ni Mouzone n'en font, à l'évidence, une question d'argent : à leurs yeux, c'est bel et bien d'une affaire d'honneur qu'il s'agit. À mesure que le cadre se resserre sur lui, l'enfermant progressivement à la manière d'un étau, Bell se rend compte que rien n'entamera la détermination de ses deux assaillants et que, par conséquent, sa fin est proche (« It seem like I can't say nothing to change your minds »). Sur son visage à présent cadré en gros plan (voir figure 1), se succèdent diverses mimiques qui traduisent la résignation de l'homme se sachant condamné - ce qui ne l'empêchera toutefois pas, ultime sursaut d'orgueil, de hausser le ton une dernière fois («Well, get on with it, motherfuck... ! »), avant de succomber sous les feux croisés d’Omar et de Mouzone et d'être laissé, seul et gisant, dans une pièce à présent désertée ${ }^{12}$.

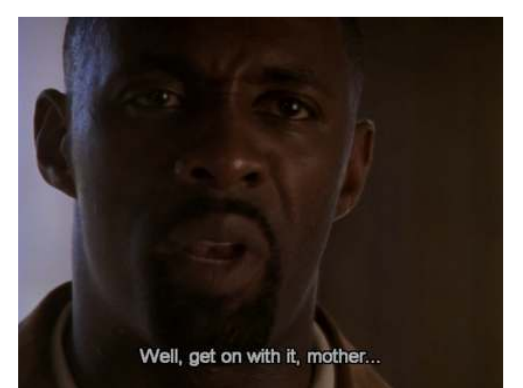

Fig. 1 : L’ultime réplique de Stringer Bell (3.11)

À cette exécution effective et armée, fait pendant, dans l'épisode suivant (3.12: "Mission Accomplished»), une exécution d'ordre symbolique quant à elle : celle dont fait l'objet le Major Howard « Bunny » Colvin. Dans le cadre de la saison 3, Colvin, pressé par sa hiérarchie, cherche par tous les moyens à faire baisser, dans le district ouest de Baltimore, le pourcentage des délits engendrés par le trafic de stupéfiants aux coins des rues. Face à une situation qu'il perçoit comme désespérée, le Major décide, à l’insu de ses supérieurs, d'instaurer trois «zones libres » à l'intérieur desquelles le trafic et la

${ }^{12}$ Dans cette séquence, certains commentateurs ont voulu voir une manière de retour de flammes. L'exécution de Stringer Bell ferait en effet écho, sous bien des aspects, à celle que lui-même avait commanditée à l'égard du jeune Wallace dans le cadre de l'épisode 1.12 («Cleaning Up »). Sur ce point, voir par exemple Stephen Lucasi, « Networks of Affiliation: Familialism and Anticorporatism in Black and White », in The Wire. Urban Decay and American Television, op. cit., p. 142 [p. 135-148]. 
consommation de drogues sont tolérés (épisode 3.4 : « Hamsterdam »). Projet audacieux et relativement efficace, mais qui, progressivement, suscitera la désapprobation des policiers de terrain. Excédé, l'un d'entre eux, Thomas "Herc » Hauk, finira par vendre la mèche en passant un coup de fil au Baltimore Sun et en invitant un journaliste incrédule à venir visiter les trois zones libres, devenues autant de modernes Cours des miracles (épisode 3.9: "Slapstick »).

Pour le projet « Hamsterdam », c'est bien évidemment le début de la fin. Certes, les coins de rue ont recouvré leur calme et les statistiques criminelles affichent une baisse significative, ainsi que Colvin le démontre à William Rawls et Ervin Burrell, ses supérieurs hiérarchiques, à l'occasion de la traditionnelle réunion hebdomadaire (épisode 3.10 : "Reformation ») - mais c'est au prix d'un grave contournement de la loi (Rawls : "Don't you see what he's done ? He legalized drugs!»). Pour Colvin, les conséquences ne se feront pas attendre. Vers la fin de l'épisode 3.12, le Major fera en effet les frais de son initiative au cours d'une véritable séance d'humiliation publique, non seulement devant ses supérieurs, mais aussi sous les yeux de ses collègues. Après avoir énuméré les multiples fautes professionnelles imputables à Colvin, Rawls y va d'une dernière provocation ("Not much you can say, is there ? Not to real police ») - assertion empreinte de mépris à laquelle le Major répond en gros plan, sur un ton à la fois décidé et dégoûté : "Get on with it, motherfucker ", avant de quitter, seul, la salle quelconque et aseptisée qui aura abrité la scène de sa destitution (voir figure 2).

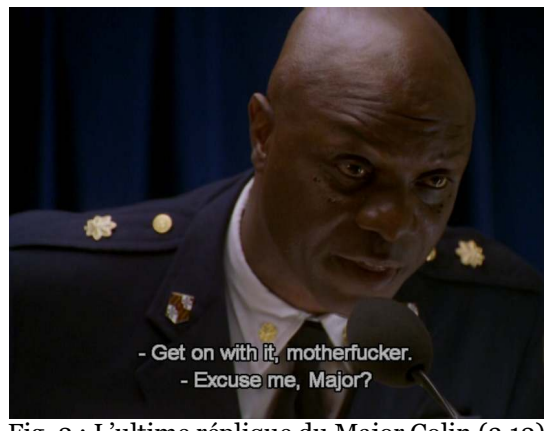

Fig. 2 : L’ultime réplique du Major Colin (3.12)

Exécution symbolique - équivalant, pour l'intéressé, à une perte de grade - mais exécution tout de même et non moins sommaire, en cela pas très éloignée de celle que dut subir Stringer Bell dans le cadre de l'épisode précédent. Une fois encore, de singuliers parallélismes se font jour d'épisode à épisode : de part et d'autre, c'est un homme seul (Bell/Colvin) qui endosse le statut de victime face à deux bourreaux 
(les duos Omar/Mouzone et Burrell/Rawls, respectivement) ; de part et d'autre, c'est la victime qui exhorte ses bourreaux à ne pas faire durer le plaisir, en usant d'une injonction et d'une insulte en tous points identiques, effet de réplique au travers duquel se suggère cette fois une possible homologie entre les deux personnages, disposés symétriquement des deux côtés d'une barrière nommée Loi ${ }^{13}$.

Comme fait d'intratextualité, la réplique constitue un procédé d'écriture que The Wire exploite en tous sens et tout au long de ses cinq saisons. Pour s'en convaincre, il suffira de se projeter, au prix d'un nouveau bond dans le temps, dans la quatrième saison de la série et de se pencher plus spécifiquement sur son deuxième épisode («Soft Eyes »). Une première actualisation de répliques en écho se joue autour des personnages respectifs de Dennis « Cutty » Wise et de Bubbles, le vagabond magnifique, Charlot des temps post-modernes.

Repris de justice, ancien bras armé du clan Barksdale, Dennis Wise a trouvé du travail dans une petite entreprise de jardinage à domicile, où il côtoie essentiellement des collègues mexicains, eux aussi passés par la case " prison ». Au début de l'épisode 4.2, une brève séquence nous montre Wise au travail, alors qu'il adresse quelques mots en espagnol à ses collègues sud-américains. Intrigué par cette compétence linguistique, le patron de Wise lui adresse la proposition suivante : "Si on faisait équipe, on pourrait couvrir deux fois plus de terrain et gagner deux fois plus » (« [...] if we put in together, we could be coverin' twice the ground, makin' twice as much ») (voir figures 3 et 4). Flatté par cette proposition, Wise n'y donnera cependant pas suite, prétextant d'autres obligations (nommément, la gestion de sa salle de boxe).
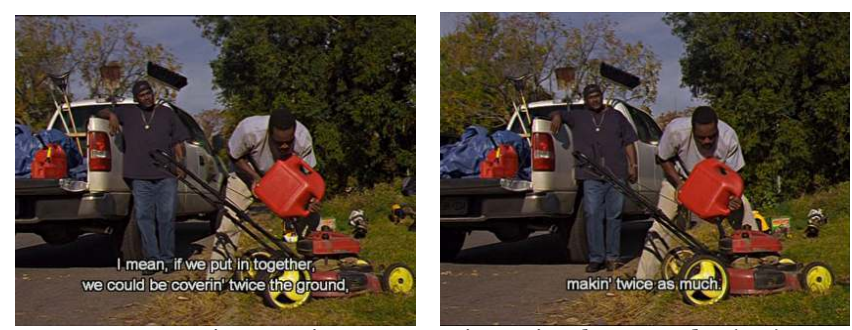

Fig. 3 et Fig. 4 : « covering twice the ground » (4.2)

${ }^{13}$ Sur ce point, voir par exemple Kieran Aarons et Grégoire Chamayou, " Contradictions », in The Wire. Reconstitution collective, op. cit., p. 65-87. Selon ces deux auteurs, les personnages de Colvin et de Bell se ressemblent en ce qu'ils sont essentiellement des " réformateurs », instigateurs d’utopies « développée[s] en miroir » (p. 73). 
Plus loin dans le même épisode, un plan-séquence long d'une minute environ nous montre, en plan large, Bubbles arpentant une ruelle avec sa boutique ambulante («Bubble's Depo »), en compagnie de Sherrod, un adolescent déscolarisé qu'il a pris sous son aile. En substance, la discussion tourne autour des lacunes du jeune homme en matière d'arithmétique élémentaire et de leurs répercussions négatives sur la petite entreprise que Bubbles porte littéralement à bout de bras. La situation incite ce dernier à placer Sherrod face à ses responsabilités: "Si je pouvais te laisser gérer l'argent seul, on pourrait couvrir deux fois plus de terrain et vendre deux fois plus » ( «If I can cut you loose to handle the money, we can cover twice the ground, selling twice the merchandise ») (voir figures 5 et 6). Moins de dix minutes séparent les deux occurrences, et l'effet d'écho s'y fait d'autant plus sensible que les phrases invoquées, circulant dans les deux cas de l'employeur vers l'employé, obéissent à la même structure grammaticale (une proposition conditionnelle, une proposition principale scandée par l'adverbe «twice » - lequel, éloquemment, dit deux fois la répétition) et répondent au même principe, celui, à la fois, d'une collaboration et d'une possible extension en termes de « parts de marché » (comme le dira Bubbles peu après), et cela au moment même où les barons de la drogue ont décrété que, pour leur part, le produit devait désormais primer sur le territoire ${ }^{14}$.

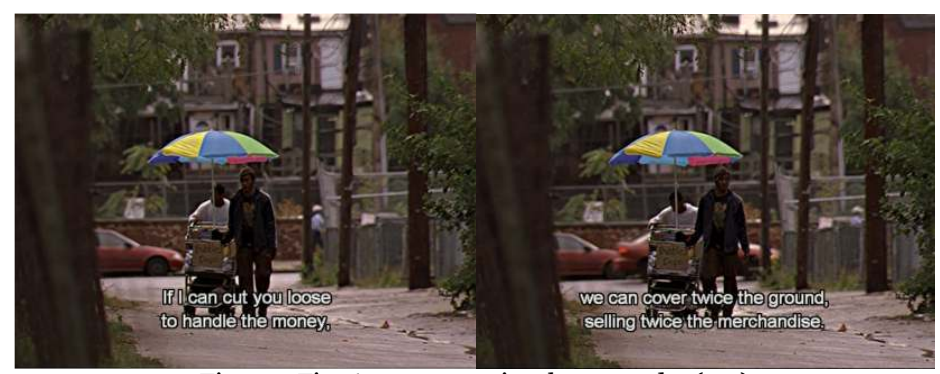

Fig. 5 et Fig. 6 : « cover twice the ground » (4.2)

${ }_{14}$ Tel est bien le sens du véritable credo que le personnage de Stringer Bell, probablement inspiré par les cours de macro-économie qu'il suit assidûment au Community College de Baltimore, prononce devant ses ouailles au début de l'épisode 3.1: "On arrête de se préoccuper du territoire, des rues ou des cités qui nous appartiennent. Le trafic, ce n'est plus ça : ce qui compte, c'est le produit » («We're done worrying about territory, man, what corner we got, what project. Game ain’t about that no more : it's about product »). L'on sait par ailleurs que cette approche réformée et en quelque sorte pacifiée du trafic de stupéfiants sera à l'origine de dissensions de plus en plus profondes entre Stringer Bell et son " patron » Avon Barksdale. Sur les postulats qui informent la prise de position de Bell, voir notamment Jason Read, "Stringer Bell's Lament: Violence and Legitimacy in Contemporary Capitalism ", in The Wire. Urban Decay and American Television, op. cit., p. 122-134. 
Cet effet de rime interne tend à indiquer qu'entre le patron de Dennis Wise et Bubbles, les situations sont homologues à bien des égards - indice ponctuel, mais particulièrement significatif, de ce que ces destins sont, d'une façon ou d'une autre, liés, connectés à distance, sans que ces personnages se croisent effectivement dans la trame du récit ${ }^{15}$.

Dans le cadre du même épisode 4.2, un autre effet de réplique se laisse repérer, légèrement plus complexe et plus audacieux quant à lui, dans la mesure où il convoque deux sphères sociales que tout semble devoir opposer. Juste après le plan-séquence mettant en scène la discussion entre Bubbles et Sherrod, le récit nous transporte sur le seuil d'une maison, où conversent notamment trois des quatre adolescents dont la quatrième saison de The Wire suit les trajectoires contrastées. C'est la fin de l'été. La rentrée scolaire n'est pas loin, et les personnages respectifs de Namond Brice, Randy Wagstaff et Michael Lee envisagent cet événement avec une certaine impatience. Ces bavardages innocents sont cependant interrompus par l'entrée en scène de Monk, premier lieutenant du dealer en chef Marlo Stanfield. Dépêché par son patron, Monk vient distribuer de l'argent liquide à ce groupe d'adolescents, façon d'acheter leur silence et/ou leur complicité. Souvent présenté comme le membre le plus indépendant du groupe, Michael refuse cet argent, ce qui provoque la stupéfaction de Marlo Stanfield en personne, lequel traverse la rue pour venir l'interpeller, occasion d'un champ/contrechamp particulièrement intense. Momentanément suspendue, la séquence reprend quelques minutes plus tard. En travelling latéral, puis arrière, la caméra suit les personnages de Namond, Randy et Michael alors qu'ils déambulent dans les rues, évoquant l'argent distribué par le clan Stanfield. Si Michael a refusé, c'est, dit-il, parce qu'il n'aime pas être redevable envers qui que ce soit ("That owin' niggas for shit, man. That ain't me »). Moins scrupuleux, Namond - pourtant le plus favorisé des trois adolescents en termes de capital matériel - résume quant à lui sa position en une seule phrase : «Je prendrai toujours son foutu blé s'il a envie de le distribuer » ( « I'll take any motherfuckin' money if he givin' it away now ») (voir figures 7 et 8 ).

\footnotetext{
15 D'un autre point de vue, ce phénomène d'écho nous renseigne tout autant sur la dimension quasiment structuraliste de l'écriture développée par Simon et Burns que sur les effets de symétrie qu'elle cultive volontiers. Dans le cas de Dennis Wise, c'est ainsi une compétence qui est pointée du doigt par l'employeur alors que, dans le cas de Sherrod, c'est précisément une déficience ; dans le premier cas de figure, la compétence est en outre de nature linguistique, alors que, dans le second, elle est précisément d'ordre arithmétique.
} 


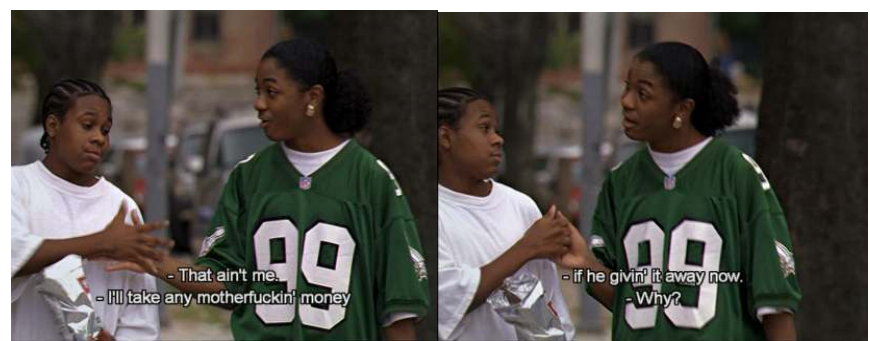

Fig. 7 et Fig. $8:$ « giving it away » (4.2)

Indice de la cupidité du personnage, cet énoncé, plutôt banal en apparence, fera retour quelques minutes plus tard, en un contexte tout différent, à hauteur d'une des autres grandes lignes narratives explorées par cet épisode. Alors que l'étau se resserre autour de sa personne et qu'il vient de recevoir une très officielle assignation à comparaître, le sénateur corrompu Clay Davis, dont la première saison de The Wire a déjà pris soin de détailler la vénalité, entend exprimer son courroux au maire Clarence Royce, principal bénéficiaire de ses malversations financières. Au beau milieu de son plaidoyer pro domo, sous le regard résigné du maire, Davis pose à son tour, sur un mode presque oratoire, la question de la provenance de l'argent : « Tu crois que j'ai le temps de demander à un mec pourquoi il me donne de l'argent? Ou bien d'où il provient ? Je prendrai l'argent de n'importe quel enfoiré s'il a envie de me le donner » ("You think I have time to ask a man why he givin' me money? Or where he gets his money from ? I'll take any motherfucker's money if he givin' it away ») (voir figures 9 et 10).

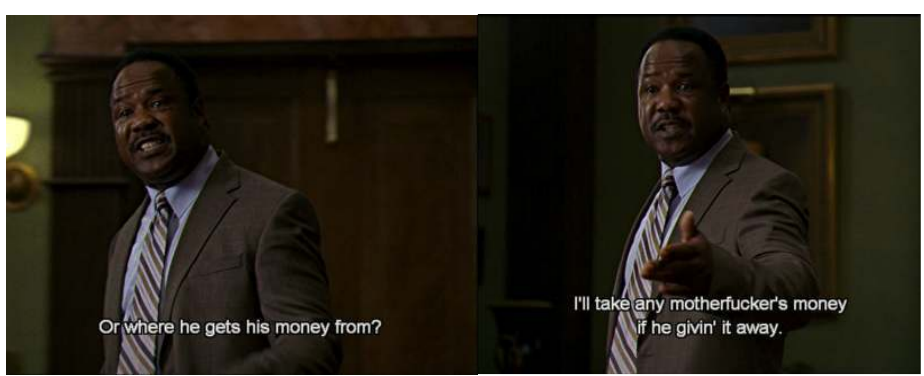

Fig. 9 et Fig. 10 : « giving it away » (4.2)

Nouvelle itération, nouvel effet de réplique. Qu'il s'agisse de Namond Brice ou de Clay Davis - deux personnages qui, à nouveau, ne se croiseront jamais dans l'espace du récit -, le principe est le même : peu 
importe l'origine effective de l'argent, pour autant qu'il puisse être mis à profit par son destinataire (en vue d'acheter des vêtements dans le chef de l'adolescent, en vue de financer des campagnes électorales dans celui du sénateur). Entre la culture de la rue et les coulisses de la scène politique, The Wire met de la sorte en relief des analogies, au travers d'échos ou de reprises dans les actes de parole endossés par les différents protagonistes. C'est là une autre façon, pour la série, de traiter du tissu urbain en termes d'interconnexions, même si celles-ci sont peu apparentes au premier abord. Dans The Wire, l'acte de parole lui-même est ainsi pensé dans sa dimension de réseau.

Telle serait peut-être, incarnée dans le procédé de la réplique, une quatrième fonction du langage dans The Wire: marqueur d'homologies entre les différents univers que la série entend passer au crible. Sous cet angle, la réplique dit bien le caractère profondément systémique de la perspective déployée par David Simon et Ed Burns un caractère systémique qui n'est d'ailleurs pas sans évoquer, dans ses objets comme dans sa méthode, la grande tradition de l'anthropologie urbaine américaine (William Foote Whyte, Elliot Liebow, etc.), à l'aune de laquelle la série gagnerait assurément à être lue, notamment dans une visée généalogique ${ }^{16}$.

Fait assez remarquable de ce point de vue et occasion d'une ouverture finale : dans la post-face de The Corner. A Year in the Life of an Inner-City Neighbourhood ${ }^{17}$, l'étude que Simon et Burns ont publiée en 1997 au terme d'une année passée à observer le trafic de stupéfiants tel qu'il se déroulait alors, en plein jour, au coin de Fayette Street et de Monroe, les deux auteurs se réclament explicitement d'Elliot Liebow et de son ouvrage le plus célèbre, Tally's Corner, paru en 1967 et disponible depuis peu en traduction française ${ }^{18}$. Issu d'une thèse de doctorat soutenue à la Catholic University of America, Tally's Corner est une étude d'ethnographie urbaine consacrée à une communauté noire américaine dans un quartier défavorisé de Washington, au départ d'un travail de terrain effectué entre janvier 1962 et juillet 1963. De toute évidence, il y aurait une riche réflexion à mener autour des multiples homologies qui semblent devoir unir les démarches adoptées respectivement par Liebow et par le tandem Simon/Burns. En tout état de cause, The Corner, comme œuvre de narration non-fictionnelle, mais aussi comme mini-série (HBO, 2000),

${ }^{16}$ Sur ce point, voir notamment Linda Williams, On The Wire, Durham, Londres, Duke University Press, 2014 et, plus particulièrement, le premier chapitre intitulé « Ethnographic Imagination. From Journalism to Television Serial », pp. 11-36.

17 David Simons et Ed Burns, The Corner. A Year in the Life of an Inner-City Neighbourhood, Edimbourg, Londres, New York, Melbourne, Canongate, 2009, p. 614

${ }_{18}$ Tally's Corner. Les Noirs du coin de la rue, Rennes, Presses Universitaires de Rennes, 2010 - avec une très intéressante introduction de la part de Célia Bense Ferreira Alves, la traductrice. 
semble marquer la gestation d'un véritable regard anthropologique qui informera en sous-main tout le propos de The Wire. Comme microphénomène, la réplique pourrait constituer la manifestation exemplaire de ce regard immergé, attentif aux moindres détails (homologies, modulations, échos, etc.).

\section{Bibliographie}

Aarons Kieran, Chamayou Grégoire, "Contradictions", The Wire. Reconstitution collective, Emmanuel Burdeau et Nicolas Vieillescazes (éds.), Paris, Les Prairies Ordinaires/Capricci, 2011, p. 65-87.

Alvarez Rafael, The Wire. Truth Be Told, Edimbourg, Londres, New York, Melbourne, Canongate, 2009, p. 246-250.

Coulouma Flore, "'That's the game, yo': Stereotype and Identity in The Wire », TV/ Series, No. 1, juin 2012, p. 144-162.

KINDER Marsha, "Re-Wiring Baltimore: The Emotive Power of Systemics, Seriality, and The City », Film Quarterly, Vol. 62, No. 2, hiver 2008-2009, p. 50-57.

Liebow Elliot, Tally's Corner: A Study of Negro Streetcorner Men, Maryland, Rowman \& Littlefield Publishers, 1967, 2003 ( $2^{\text {nd }}$ edition).

Liebow Elliot, Tally's Corner. Les Noirs du coin de la rue (1967), trad. Célia Bense Ferreira Alves, Rennes, Presses Universitaires de Rennes, 2010.

LUCASI Stephen, «Networks of Affiliation: Familialism and Anticorporatism in Black and White ", The Wire. Urban Decay and American Television, éd. Tiffany Potter et C. W. Marshall, New York, Londres, Continuum, 2009, p. 135-148.

MitTell Jason, « Narrative Complexity in Contemporary American Television ", The Velvet Light Trap, No. 58, hiver 2006, p. 29-40.

NANNICELLI Ted, «It's All Connected: Televisual Narrative Complexity ", The Wire. Urban Decay and American Television, Tiffany Potter et C. W. Marshall (éds.), New York, Londres, Continuum, 2009, p. 190-202.

Potte-Bonneville Mathieu, "All in the game», The Wire. Reconstitution collective, Emmanuel Burdeau et Nicolas Vieillescazes (éds.), Paris, Les Prairies Ordinaires/Capricci, 2011, p. 147-166. 
READ Jason, «Stringer Bell's Lament: Violence and Legitimacy in Contemporary Capitalism ", The Wire. Urban Decay and American Television, op. cit., p. 122-134.

Simon David, Burns Ed, The Corner. A Year in the Life of an InnerCity Neighbourhood, Edimbourg, Londres, New York, Melbourne, Canongate, 2009.

Williams Linda, On The Wire, Durham, Londres, Duke University Press, 2014.

\section{L'auteur}

Livio Belloï est chercheur qualifié du Fonds National de la Recherche Scientifique (Bruxelles) et Maître de conférences à l'université de Liège. Ses recherches portent essentiellement sur le cinéma des premiers temps, sur le cinéma expérimental (Brakhage, Deutsch, Morrison, Sharits, etc.), sur les séries télévisées américaines et sur la bande dessinée expérimentale contemporaine (cf. http://orbi.ulg.ac.be/simple-search?query=belloi). Ses deux derniers ouvrages s'intitulent Film ist. La pensée visuelle selon Gustav Deutsch (Lausanne, L'Âge d'Homme, coll. " Histoire et esthétique du cinéma », 2013) et La Mécanique du détail. Approches transversales (Lyon, ENS Editions, coll. "Signes », 2014, en co-direction avec Maud Hagelstein). 